\title{
Novel dual single sided silicon strip detector chip for radiotherapy verification
}

\author{
M.A.G. Alvarez* \\ Author affiliation: Instituto de Física da Universidade de São Paulo (IFUSP), 05508-090, São \\ Paulo, Brazil. \\ E-mail: malvarez@us.es
}

\author{
Z. Abou-Haïdar ${ }^{a}$, R. Arráns ${ }^{c}$, M.C. Battaglia ${ }^{a}$, M.A. Cortés-Giraldo ${ }^{b}$, J.M. Espino $^{a, b}$, \\ M.I. Gallardo ${ }^{b}$, M.C. Ovejero ${ }^{b}$, A. Pérez-Vega Leal ${ }^{d}$, A. Selva ${ }^{b, f}$ and S. Walsch ${ }^{e}$ \\ Affiliation: a) Centro Nacional de Aceleradores (CNA) de la Universidad de Sevilla, Junta de \\ Andalucía y CSIC, 41092, Seville, Spain \\ b) Departamento de Física Atómica, Molecular y Nuclear (FAMN) de la Universidad de Sevilla, \\ 41013, Seville, Spain \\ c) Hospital Universitario Virgen Macarena, 41071, Seville, Spain \\ d) Escuela Superior de Ingenieros de la Universidad de Sevilla, 41092, Seville, Spain. \\ e) Micron Semiconductor Ltd., Sussex BN15 8UN, England, United Kingdom. \\ f) Dipartimento di Fisica e Astronomia 'Galileo Galilei', 35131, Padova, Italy
}

\begin{abstract}
A novel dual single sided silicon strip detector (SSSSD) chip was designed to meet clinical requirements in radiotherapy verification. An available design from Micron Semiconductor Ltd. (BB7, $500 \mu \mathrm{m}$ thick) was the base of a two-dimensional detector adapted into a special configuration with the aim of uniforming and minimizing foreing materials around the active area $\left(64 \times 64 \mathrm{~mm}^{2}\right)$. With this purpose, two independent BB7 SSSSDs were mounted in a perpendicular configuration, separated by a $500 \mu \mathrm{m}$ kapton dielectric film with the same dimensions as the silicon wafers, thus minimizing air gaps in between. This new configuration, called the dual SSSSD chip design, was mounted on kapton printed circuit board (PCB). Both silicon wafers were divided into 32 strips, $2 \mathrm{~mm}$ width each. The aim of developing this detector was to allow 2D dose measurements, improve spatial resolution and perform radiotherapy treatment verification faster than with a previous prototype. Characteristics and performance of the novel detector are presented.
\end{abstract}

X Latin American Symposium on Nuclear Physics and Applications (X LASNPA),

1-6 December 2013

Montevideo, Uruguay

${ }^{*}$ Speaker. 


\section{Introduction}

Many efforts have been done to develop silicon detectors for medical applications, taking advantage of the experience and advances earned in detector technology from nuclear physics research (i.e. tracking silicon detectors, electronics and data acquisition systems) [1]. In particular, research is oriented towards silicon strip/pixel technology to improve spatial resolution for complex radiotherapy verification $[2,3]$. As a matter of fact, silicon pixel detectors were developed to obtain good spatial resolutions, such as MAESTRO, DOSI, and CMRP DMG detectors [4, 5, 6, 7, 8, 9]. They require a high number of allocated channels and complex multichannel readout electronics based on application specific integrated circuits (ASIC) coupled directly to the detection system. This can produce some inconveniences due to the sensitivity of ASICs to radiation. In addition, these systems are not suitable to obtain dose maps in axial planes, which are generally oriented parallel to the beam axis. In fact, many of them are designed to be irradiated only perpendicularly with respect to detector active area. This means that they are useful for verifying separately each treatment field but not the full plan.

Within this scenario, we developed a novel solution and studied its feasibility to obtain twodimensional (2D) axial dose maps in radiotherapy treatments using a single sided silicon strip detector (SSSSD) [10, 11, 12]; concretely, we used the 16-strip design W1-SS 500 from Micron Semiconductor Ltd. [13] The detector was placed at the axial plane of a cylindrical rotating phantom and the whole system was irradiated under clinical conditions by a Siemens linear accelerator (linac) installed at the Virgen Macarena Hospital in Seville (Spain). After successful results using the W1-SS 500, we present the development of an advanced prototype. The aim is to improve the traditional devices and to verify in a simple, fast and correct way, a complete radiotherapy treatment.

\section{The Detector}

The new detector concept was designed, developed and built within the context of RADIA collaboration (University of Seville, the National Accelerator Centre (CNA) and Virgen Macarena Hospital), with the support of the companies ATI sistemas S. L. and Micron Semiconductor Ltd. [13]; the latter was also responsible for its construction. Characteristics of the W1-SS 500 detector, used previously, are summarized in references $[10,13]$. The detectors are shown in figure 1 . In the new design, based on the same silicon strip technology, we decided to modify four key elements for improving complex radiotherapy treatment verification:

1) Bigger active area $\left(\mathbf{6 4 \times 6 4} \mathbf{~ m m}^{2}\right)$ to allow measurements of larger dose maps.

2) Smaller strip width $(\mathbf{2} \mathbf{~ m m})$, i.e. 32 strips per wafer, to improve the spatial resolution.

3) Two perpendicular strip orientations to allow simultaneous measurements in two different orientations, hence reducing considerably the acquisition time.

4) The packaging of the detector, minimizing it and improving the density uniformity of materials with respect to the previous design.

We chose an already existing silicon wafer model from Micron Semiconductor Ltd., usually employed for the manufacturing of the $500 \mu \mathrm{m}$ single sided BB7 model. In addition, we wanted to 
implement the ability to perform simultaneous measurements with two different strip orientations. Due to this assembly, we have called this new detector the dual SSSSD chip design.
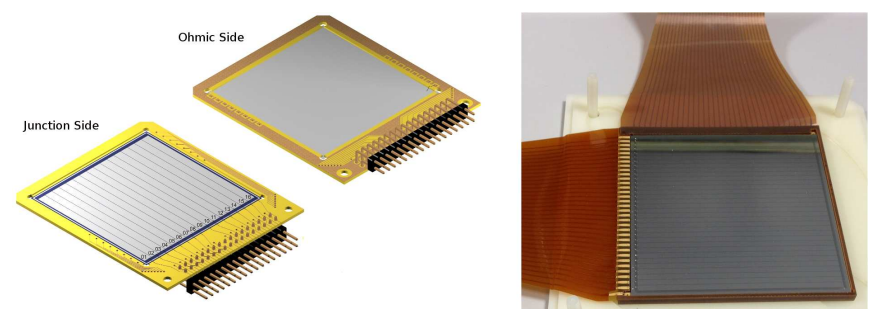

Figure 1: Single sided silicon strip W1-SS 500 model detector (on the left) provided by Micron Semiconductor Ltd.[13], compared to the novel dual SSSSD chip design (on the right).

Another goal of the new setup is to minimize the perturbation due to placing the detector in the medium (similar to water) where absorbed dose is to be measured. This can be achieved by uniforming detector composition with material densities as close as possible to $1 \mathrm{~g} / \mathrm{cm}^{3}$ (water density). Thus, for the frame of the new detector $\left(67 \times 67 \mathrm{~mm}^{2}\right)$, we chose $1200 \mu \mathrm{m}$ Kapton (Arlon $85 \mathrm{~N}$ polyimide laminate from DuPont), with a density of $1.6 \mathrm{~g} / \mathrm{cm}^{3}$. Besides, the frame dimensions were decreased from $\sim 1 \mathrm{~cm}$ to $1.5 \mathrm{~mm}$. Since Kapton presents very low thermal conductivity and very good insulating properties, we used a $500 \mu \mathrm{m}$ Kapton film as dielectric separator between both silicon wafers, avoiding air gaps between them. Following the idea of minimizing foreign material around the detecting volume, we replaced the pin connector found on the W1-SS 500 design by two $200 \mathrm{~mm}$ length thin flat Kapton cables; at the end of which we installed pin connectors for the strips (Samtec TSW-117-7-L-D), thus keeping away the copper connectors from the detector (fig. 1). The flat cables directly connected to the silicon strips are made of $50 \mu \mathrm{m}$ DuPont AP8525R $\left(\rho=1.43 \mathrm{~g} / \mathrm{cm}^{3}\right)$ core, with $18 \mu \mathrm{m}$ of copper on each side $\left(\rho=8.2 \mathrm{~g} / \mathrm{cm}^{3}\right)$.
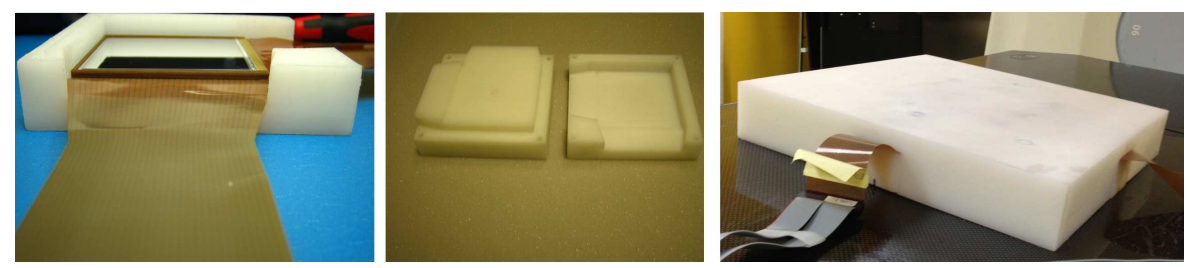

Figure 2: The final version of the dual SSSSD chip (on the left) for radiotherapy verification, placed in a polyethylene box (in the middle), which is designed to be coupled to different phantoms (on the right).

In summary, our dual SSSSD chip prototype consisted of the two SSSSDs with serial numbers 2958-2 (BB7-SS with $500 \mu \mathrm{m}$ thickness) and 2958-6 (BB7-SS, $504 \mu \mathrm{m}$ ), mounted together (separated by Kapton as explained above) with the detector strips perpendicular to each other. The prototype was installed in a polyethylene box, for protection, before being placed in a slab phantom (fig. 2) for the first measurements.

\section{Measurements}

The system was irradiated in the Radiotherapy Unit of Virgen Macarena Hospital (Sevilla, 
Spain) by a Siemens PRIMUS clinical linac (Figure 3) in $6 \mathrm{MV}$ photon mode. As it is well known, clinical linacs used for X-ray radiotherapy, accelerate electrons which generate bremsstrahlung radiation when colliding with a tungsten target placed at the beam entrance of the treatment head. Typical energies of the primary electron beam are $6 \mathrm{MeV}$ and $15 \mathrm{MeV}$ (commonly referred to as $6 \mathrm{MV}$ or $15 \mathrm{MV}$ mode). The photon fields are shaped by means of a multileaf collimator (MLC) moving along the $\mathrm{X}$-axis, and jaws moving along the $\mathrm{Y}$-axis (provided the radiation beam axis is $\mathrm{Z}$ axis), both systems installed within the linac treatment head. At therapy energies, photons interact with water equivalent material of the phantoms (or the human body tissue) mainly by Compton interaction releasing electrons into medium. As these electrons travel through the detector, they lose kinetic energy by exciting and ionizing atoms of the material, creating carriers which generate a charge (or voltage) output. In order to validate and characterize the system as a dosimeter for radiotherapy irradiations one, first of all, has to find a relation between this output and the dose absorbed in the medium if the detector were not present.

To do that, we have performed several measurements with the detector hosted inside a slab phantom and irradiated with the beam axis perpendicular to its active area (Figure 3), following closely a clinical protocol for quality assurance purposes.

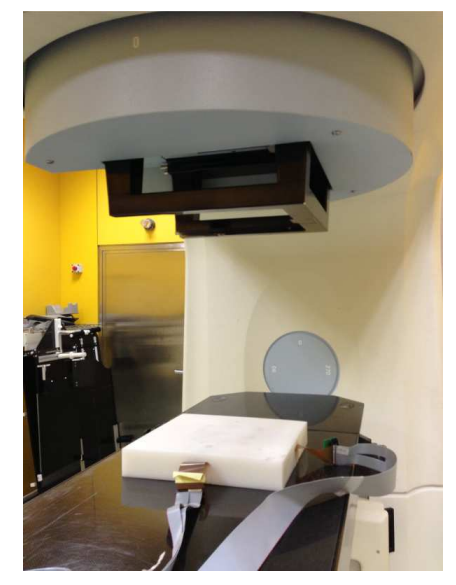

Figure 3: Dual SSSSD chip placed into a slab phantom and positioned on the treatment couch to be irradiated by the linac at the Hospital.

Fig. 4 shows preliminary results of the detector response characterization:

1. Plot (a) shows the behavior of one detector strip under irradiation as a function of time. The strip collects the electrons generated by Compton scattering with a linear response that allows to calibrate the system as a dosimeter.

2. In plot (b), the response of individual strips when irradiated by a uniform field is represented. Different responses of the upper and lower detector can be observed. The red points represent the raw signal from each strip, while the blue ones show the average response of each detector, that leads to the uniformity factor calculation.

3. The study of the percentage depth dose is shown at (c). The results of the dose deposited at 10 and $15 \mathrm{~cm}$ in solid water depth (red points) are compared with those measured by an ion- 
ization chamber (blue dots) at Virgen Macarena Hospital under clinical reference conditions $\left(10 \times 10 \mathrm{~cm}^{2}\right.$ field, water phantom surface at the isocenter plane).

4. Plot (d) shows the penumbra, defined as the region located between $20 \%$ and $80 \%$ of the maximum dose level, along the $\mathrm{X}$ (red squares) and $\mathrm{Y}$ (blue squares) directions. This study has been carried out by irradiating the system with asymmetric fields of $5 \times 10 \mathrm{~cm}^{2}$ and $10 \times 5 \mathrm{~cm}^{2}$ respectively.
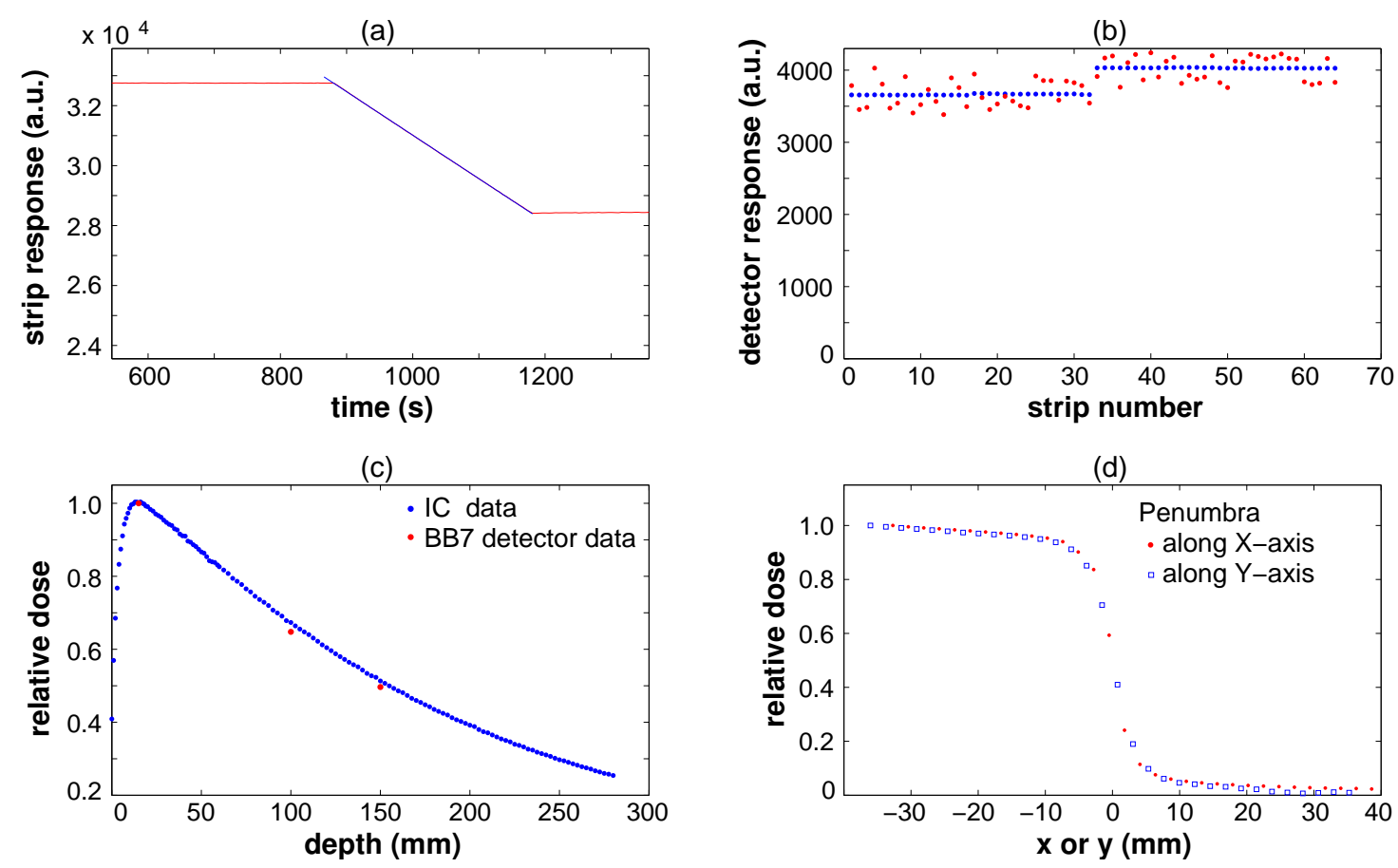

Figure 4: Preliminary tests of the detector responses in terms of: silicon strip uniformity, linearity, PDD and penumbra.

\section{Acknowledgments}

This project was partly supported by the specific collaboration agreement, under reference 68/63 OG-049/07, signed between the "Fundación de Investigación de la Universidad de Sevilla" (FIUS) and "Instalaciones Inabensa S. A." company, to finance the project RADIA, under reference in FIUS, CP:0214, CGT:0129. The research is also partly funded by the Spanish Ministry of Science and Innovation under contracts FPA2009-08848, FPA2011-28770-C03-02 and FIS201128738-C02-01 and by the Council for Innovation, Science and Commerce from the Andalusian Government, contracts P11-FQM-7632 and P12-FQM-1605. The authors would like to thank Mrs. Marta Trueba in representation of "ATI Sistemas S. L." company. This work is also partly financed by the specific collaboration agreement, under references CP:1751, CGT:0636, signed between FIUS and "ATI Sistemas S. L." . M. C. Battaglia would like to thank the "optimization of particle accelerators" (oPAC) project, supported by the European Union (EU), under reference PITN-GA2011-289485. 


\section{References}

[1] R. Ballabriga, et el., Nucl. Instrum. Methods Phys. Res., Sect. A. 633 (2011) S15-S18.

[2] I. Redondo-Férnandez, et al., Nucl. Instrum. Methods Phys. Res., Sect. A. 573 (2007) 141-144.

[3] A. J. Cullen, Master's thesis, Centre for Medical Radiation Physics-Faculty of Engineering, University of Wollongong, 2009.

[4] C. Talamonti, et el., Nucl. Instrum. Methods Phys. Res., Sect. A. 583 (2007) 114-118.

[5] S. Manolopoulos, et al., Physics in Medicine and Biology 54 (2009) 485.

[6] D. Menichelli, et al., Nucl. Instrum. Methods Phys. Res., Sect. A. 583 (2007) 109-113.

[7] J. L. Bedford, et al., Physics in Medicine and Biology 54 (2009) N167.

[8] R. Sadagopan, et al., Journal of Applied Clinical Medical Physics 10 (2009) 2928.

[9] C. Talamonti, et al., Nucl. Instrum. Methods Phys. Res., Sect. A. 658 (2011) 84.

[10] A. Bocci, et al., Nucl. Instrum. Methods Phys. Res., Sect. A. 673 (2012) 98-106.

[11] Z. Abou-Haidar, et al., Phys. Rev. ST Accel. Beams 15 (2012) 042802.

[12] M. A. Cortés-Giraldo, et al., Progress in Nuclear Science and Technology 2 (2010), 191-196

[13] Micron Semiconductor Ltd, Solid State Detectors and Internet Solutions, Available on-line at http://www.micronsemiconductor.co.uk/ (2013). 